\title{
Activation of a system A amino acid transporter, ATA1/SLC38A1, in human hepatocellular carcinoma and preneoplastic liver tissues
}

\author{
NOBUO KONDOH ${ }^{1}$, NOBUO IMAZEKI ${ }^{2}$, MASAAKI ARAI ${ }^{1}$, AKIYUKI HADA $^{1}$, KAZUO HATSUSE $^{3}$, \\ HIROTAKA MATSUO $^{4}$, OSAMU MATSUBARA ${ }^{2}$, SHURI OHKURA ${ }^{1}$ and MIKIO YAMAMOTO ${ }^{1}$ \\ Departments of ${ }^{1}$ Biochemistry, ${ }^{2}$ Pathology, ${ }^{3}$ Surgery, ${ }^{4}$ Integrative Physiology and Bio-Nano Medicine, \\ National Defense Medical College, 3-2 Namiki, Tokorozawa-shi, Saitama 359-8513, Japan
}

Received January 22, 2007; Accepted March 5, 2007

\begin{abstract}
The expression of amino acid transporter (AT) mRNAs including A system (ATA1/SNAT1/SLC38A1, ATA2/ SNAT2/SLC38A2 and ATA3/SNAT3/SLC38A4), L system (LAT1/SLC7A5 and LAT2/SLC7A8), and $\mathrm{y}^{+}$(CAT2/SLC7A2) genes, were compared among hepatocellular carcinoma (HCC) and non-cancerous liver cells. Among them the ATAl mRNA expression was significantly elevated in all HCC cell lines (HepG2, HLF, HuH7 and JHH4) examined compared with normal liver tissue. We further discovered that the expression of ATAl mRNA was significantly activated in HCC tissues and also elevated in pre-malignant cirrhotic livers from HCC patients, compared with normal livers from non-HCC patients. The ATA1 protein was extensively accumulated in the cytoplasm of pre-malignant liver and most HCCs, while being weak or undetectably low in normal liver tissues. SiRNAmediated suppression of endogenous ATAl lowered the viability of HepG 2 cells. Thus, the activation of ATA1 confers growth and survival advantages in pre-malignant and malignant liver lesions.
\end{abstract}

\section{Introduction}

Amino acids are required for a wide variety of important biological processes including malignant transformation in mammalian cells. Indeed, most cells activate various different systems that are required for amino acid transport (1). Among these systems, the system L-type amino acid transporter-1 (LAT-1) is up-regulated in human bladder carcinoma cells (2), esophageal adenocarcinoma (3), epidermoid carcinoma (4), oral squamous cell carcinoma (5) and liver cancer (6). On the other hand, another of the major amino acid transport

Correspondence to: Dr Nobuo Kondoh, Department of Biochemistry, National Defense Medical College, 3-2 Namiki, Tokorozawa-shi, Saitama 359-8513, Japan

E-mail: nkondoh@ndmc.ac.jp

Key words: hepatocellular carcinoma, amino acid transporter, ATA1, siRNA systems, system A, catalyses the transport of most of the small aliphatic amino acids including alanine, serine and glutamine (1). System A gene activity is dependent on cellular growth in many cell types, and increases in chemically and virally transformed cells (1). Moreover, physiological liver hyperplasias, including those that occur during pregnancy, lactation and fetal and neonatal development, are mainly associated with increased system A activity (1).

There are three known system A gene isoforms which primarily differ by their tissue expression patterns. ATA1 is primarily expressed in the neurons in the brain and spinal cord, but is not observed in lung, liver, spleen or kidney (7). ATA2 is ubiquitously expressed in every tissue (8), and ATA3 is expressed almost exclusively in the liver (9). Among these genes, ATA2 activity is induced in regenerating livers $(10,11)$. In carcinomas, the estrogen-dependent activation of system $\mathrm{A}$ is observed in breast cancers (12). Furthermore, inherent differences in the system A carrier are observed between normal and transformed liver tissues (13). On the other hand, L-arginine is an essential rate-limiting nutrient for the survival of different tumor cells, which cannot synthesize this amino acid (14). The $\mathrm{y}^{+}$cationic amino acid transporter genes (CAT1, 2 and 3) encode the transporters that mediate most arginine uptake in mammalian cells (1).

In our present study, we analyzed the expression of several amino acid transporter genes, including those of system L, $\mathrm{A}$ and $\mathrm{y}^{+}$in hepatocellular carcinoma cells, and our data demonstrate the physiological importance of ATA1 in hepatocarcinogenesis.

\section{Materials and methods}

Tissue specimens and HCC cell lines. Surgically resected $\mathrm{HCC}$ and adjacent non-cancerous liver tissues from 25 patients, in addition to five normal liver tissue controls, were used in the RT-PCR analyses in this study. The viral status of the $\mathrm{HCC}$ patients was classified as $15 \mathrm{HCV}$ positive, $2 \mathrm{HBV}$ positive, and 8 non-B non-C. In addition, each of our HCC patients had suffered from liver cirrhosis. For immunohistochemical analysis, we examined a different series of 15 paraffin-embedded HCC and adjacent liver tissues, and 2 normal liver tissues (Table I). All of these studies were performed with the informed consent of each patient and with the permission of the ethics committee of the National 
Table I. Immunohistochemical analysis of ATA1 in HCC and non-cancerous liver tissues.

\begin{tabular}{|c|c|c|c|c|c|}
\hline \multirow[b]{2}{*}{ Specimens } & \multirow[b]{2}{*}{ Viral status $^{a}$} & \multicolumn{2}{|c|}{ Liver histology and HCC grading } & \multicolumn{2}{|c|}{ Relative ATA1 expression } \\
\hline & & Non-cancerous ${ }^{\mathrm{b}}$ & Cancerous & Non-cancerous & Cancerous \\
\hline 1 & $\mathrm{~B}$ & $\mathrm{LC}$ & Well & + & ++ \\
\hline 2 & nd & $\mathrm{LC}$ & Well & + & ++ \\
\hline 3 & $\mathrm{C}$ & $\mathrm{LC}$ & Well & + & ++ \\
\hline 4 & nd & $\mathrm{LC}$ & Moderate & + & ++ \\
\hline 5 & $\mathrm{C}$ & $\mathrm{LC}$ & Moderate & + & ++ \\
\hline 6 & $\mathrm{~B}$ & $\mathrm{LC}$ & Well & + & + \\
\hline 7 & nd & $\mathrm{LC}$ & Well & + & + \\
\hline 8 & $\mathrm{C}$ & $\mathrm{LC}$ & Moderate & + & + \\
\hline 9 & nd & $\mathrm{LC}$ & Moderate & + & + \\
\hline 10 & $\mathrm{C}$ & $\mathrm{CAH}$ & Poor & + & + \\
\hline 11 & $\mathrm{C}$ & $\mathrm{CAH}$ & Poor & + & + \\
\hline 12 & $\mathrm{C}$ & $\mathrm{LC}$ & Well & + & \pm \\
\hline 13 & NBNC & $\mathrm{CAH}$ & Well & + & \pm \\
\hline 14 & $\mathrm{C}$ & $\mathrm{CAH}$ & Moderate & + & \pm \\
\hline 15 & B & $\mathrm{LC}$ & Moderate & + & \pm \\
\hline 16 & na & Normal liver & na &, \pm- & na \\
\hline 17 & na & Normal liver & na &, \pm- & na \\
\hline
\end{tabular}

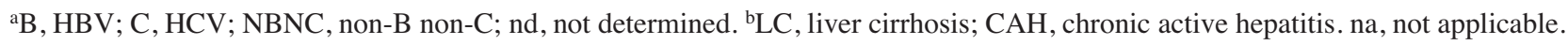

Defense Medical College. The human HCC cell lines, HLF, HuH7, JHH4 and HepG2 were obtained from the Japanese Collection of Research Bioresources (Osaka, Japan). Cells were maintained in Dulbecco's modified Eagle's minimum essential medium (DMEM) supplemented with $10 \%$ fetal bovine serum (FBS).

$R T-P C R$. RNA extraction and RT-PCR analyses were carried out in the presence of $\left[\alpha-{ }^{32} \mathrm{P}\right] \mathrm{dCTP}(2.7 \mu \mathrm{Ci}$ per sample $)$ as described previously (15), using the specific oligonucleotide primer pairs ATAl (NM_030674; 5'-CCA GTG GCC TAG CTG GTA CCA C-3' and 5'-TCC CCA GCG AAA GTT GAC TCA GAC-3'), ATA2 (AF259799; 5'-ATC TGG CTG TAA GGT TTG CCC T-3' and 5'-AAA TGG ACC AAC GGT TTC ACC C-3'), ATA3 (AF305814; 5'-ACA CTA CGA AGA AAT GCA CAC GA-3' and 5'-GCC TCC CAA TAC ATT GAG CCA-3'), LAT-1 (NM_003486; 5'-GGT CCT CGG GAT GCC GTT CT-3' and 5'-CTG AGA CAG TCC CCT GCG GT-3'), LAT-2 (NM_012244; 5'-CCC TAG AGG TGC CCA TTC CAT AC-3' and 5'-TCT GAA GGG GTC ACA AC T CAG AC-3'), and CAT-2 (NM_003046; 5'-TCC TAG CTT TCC TCG TGT TGG G-3' and 5'-TTG GGT GAT GGT CAT TTG CTT GA-3'). As an internal control, we used the $\beta_{2}$-microglobulin ( $32 \mathrm{M}$ ) primers (BC064910; 5'-ATC CAG CGT ACT CCA AAG AT-3' and 5'-TTA CAT GTC TCG ATC CCA CT-3'). Specific band intensities were quantified using BAS-2000 (Fuji film).

The expression of AT genes in HCC and non-cancerous liver tissues was examined by quantitative (Q)RT-PCR, as described previously (16). The expression values for each gene were normalized to the expression levels of the $32 \mathrm{M}$ transcript. To evaluate the statistical significance of the differentially expressed genes, a Welch's t-test (two-sided) was performed. We performed the direct sequencing analyses and observed that each PCR product was correctly amplified (not shown).

Production of polyclonal antibodies against human ATA1. An oligopeptide (DWACSSSSDEGHC) corresponding to amino acid residues $476-487$ of the human ATA1 protein was synthesized. $C$-terminal cysteine residue was used for conjugation with keyhole limpet hemocyanin. Anti-peptide antibody was then produced as described elsewhere (17) and the antiserum was affinity purified as described previously (18).

Immunohistochemical analysis. Immunohistochemical analysis of the ATA1 protein was performed using the avidin-biotinperoxidase complex method. Briefly, for antigen retrieval, the sections were treated with $0.5 \%$ trypsin at $37^{\circ} \mathrm{C}$ for $10 \mathrm{~min}$. After blocking with $1 \%$ skim milk, tissue sections were then incubated with anti-ATA1 polyclonal antibody (1:200) overnight at $4^{\circ} \mathrm{C}$, then washed in phosphate-buffered saline solution. Biotinylated anti-rabbit IgG was used as the secondary antibody. The specificity of this antibody was confirmed using blocking experiments with the ATA1 synthetic peptide.

Small interfering RNA analysis. Two pairs of anti-ATAl siRNAs were commercially obtained (Takara), 5'-GGA UGA UAA CAU UAG CAA UTT-3' and 5'-AUU GCU AAU GUU AUC AUC CTT-3' for HDCK-ATA1, and 5'-GGA 
GAU AAA GGA ACU CAA ATT-3' and 5'-UUU GAG UUC CUU UAU CUC CTT- 3 ' for DFIEX-ATA1. For transfection of these molecules, $5 \times 10^{4} \mathrm{HepG} 2$ cells were seeded in 24-well plates. One hundred pmol of specific siRNAs or randomized cocktails of dsRNA as a control was then mixed with $8 \mu 1$ of the Trans IT-TKO transfection reagent (Mirus Bio Corporation) and $53 \mu 1$ of DMEM (without FBS). After incubation for $15 \mathrm{~min}$ at room temperature, the mixture was diluted to $300 \mu 1$ with DMEM (10\% FBS) and added to the cells which were cultured for a further $12 \mathrm{~h}$. The cells were then harvested and gene expression inhibition was assessed by quantitative RT-PCR, as described previously (16), using two different ATA1-specific primer pairs $(529 \mathrm{~F} / 679 \mathrm{R}$; 5'-CAA TGA CTC CAA TGA TTT CAC CGA-3' and 5'-CAT GCC TAA GGA GGT TGT ACC TGG-3') and (1677F/1827R; 5'-TGA TCT TCA TAC CCT CCA TGA AGG-3' and 5'-GAA AAG GGC AGC CCA AAT TCT T-3').

Cell viability assay. To determine the viability of siRNAtransfected cells, the redox activity of viable cells was measured using an XTT [2,3-bis (2-methoxy-4-nitro-5-sulfophenyl)$2 \mathrm{H}$-tetrazolium-5-carboxanilide] assay. Briefly, $2 \times 10^{5}$ cells $/ \mathrm{ml}$ $(0.1 \mathrm{ml}$ aliquots) were inoculated into a 96 -well microtiter plates in triplicate. Thereafter, transfections were performed as described above except that $300 \mu 1$ of the diluted mixture was divided into triplicate wells. After 24,48 and 72 h, 50- $\mu \mathrm{l}$ aliquots of a solution containing $1 \mathrm{mg} / \mathrm{ml}$ of XTT (Sigma) and $10 \mu \mathrm{M}$ of phenazine methylsulfate (Sigma) were added to each well. The plates were then incubated for a further $2 \mathrm{~h}$ at $37^{\circ} \mathrm{C}$ to allow color development, and the absorbance was measured at $492 \mathrm{~nm}$. A relative survival ratio was calculated based on the absorbance values, measured in triplicate, from standard wells without cells.

\section{Results}

Expression of AT mRNAs in HCC cell lines. To analyze the expression of the AT genes including A system (ATAl/ SNAT1/SLC38A1, ATA2/SNAT2/SLC38A2 and ATA3/SNAT3/ SLC38A4), L system (LAT1/SLC7A5 and LAT2/SLC7A8), and $\mathrm{y}^{+}(C A T 2 / S L C 7 A 2)$ in HCC cells, we examined their expression in HCC cell lines. As shown in Fig. 1, the levels of ATAl mRNA were 15- to 30-fold higher in four HCC cell lines tested (HLF, JHH4, HuH7 and HepG2) compared with the normal liver tissue. The levels of ATA2 and LATI mRNAs were found to be moderately elevated in some of the HCC cell lines compared with the normal liver samples. The levels of CAT2 and ATA3 transcripts varied among the HCC cell lines but showed no significant differences compared with normal liver tissue. The expression of LAT2 was observed to be at almost undetectable levels both in the normal liver tissue and each of the HCC cell lines. Thus, among the ATs examined, the expression of ATAI mRNA was significantly activated in each of four separate HCC cell lines.

The expression of amino acid transporter mRNAs in normal and pre-cancerous liver tissues, and in HCC. We further examined the expression of ATA1, ATA2 and LAT1 mRNAs in 25 HCCs and pre-cancerous liver tissues from the same patients, as well as in five normal liver tissues from non-HCC

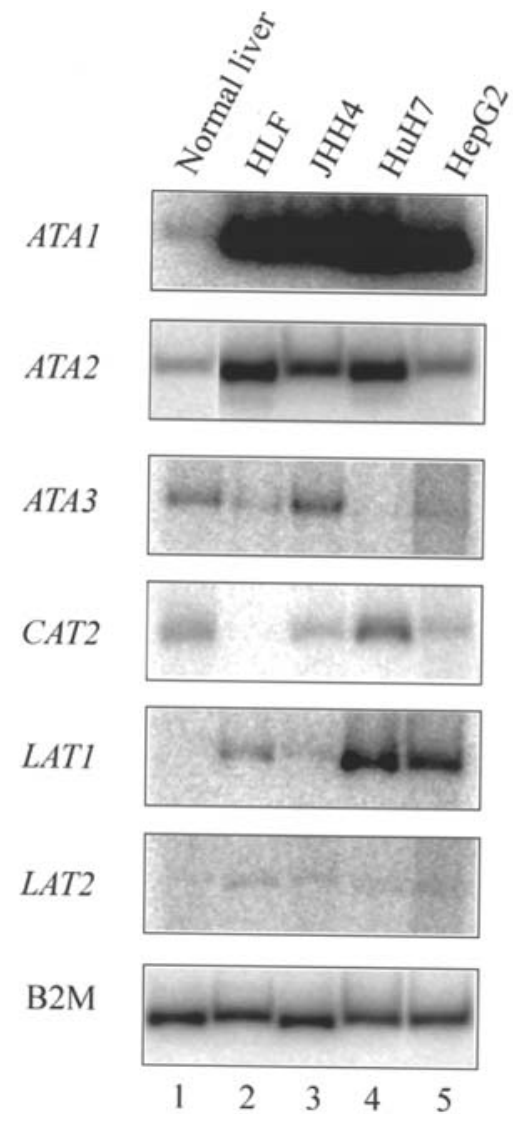

Figure 1. Expression of ATA1, ATA2, ATA3, CAT2, LAT1 and LAT2 mRNAs in the four indicated HCC cell lines and a normal liver tissue. Total RNA extracts from these tissues were subjected to RT-PCR in the presence of $\left[\alpha^{-32} \mathrm{P}\right] \mathrm{dCTP}$ for 20 cycles. The relative mRNA expression levels were then calculated by normalizing the band intensities for each gene product with a $\beta_{2}$-microglobulin control.

patients, respectively. As shown in Fig. 2, the expression of the ATAl mRNA was significantly activated in HCC tissues (average $\pm \mathrm{SE} ; 8.38 \pm 2.69$ ) compared with normal liver tissues $(1.79 \pm 1.17)$ from non-HCC patients $(\mathrm{p}=0.026)$. Interestingly, the ATA1 mRNA expression was also upregulated in non-cancerous cirrhotic livers (NLs) from HCC patients $(6.36 \pm 1.41)$; however the expression in NLs was averagely lower than in adjacent HCCs. The expression levels of the ATA2 mRNA were significantly higher in NLs $(7.44 \pm 1.39)$ than in normal livers $(2.57 \pm 0.28)$ or in HCCs (4.62 \pm 0.73$)$. On the other hand, the expression of LAT1 mRNA showed no significant difference among HCCs, precancerous and normal livers (Fig. 2). We also confirmed that the expression levels of ATAl mRNA in non-cancerous and HCC tissues were unaffected by the viral status (HCV, HBV or non-A non-B) (data not shown).

Immunohistochemical analysis of the ATA1 protein product in both non-cancerous liver and HCC tissues. To examine the expression of the ATA1 gene product in vivo, we next performed a series of immunohistochemical analyses. As shown in Fig. 3, in normal liver tissue the expression of ATA1 protein was very low or undetectable around the perivenous area, and was very weakly expressed in the cytoplasm of the parenchymal cells in the periportal area (Fig. 3A and B). 
ATA1

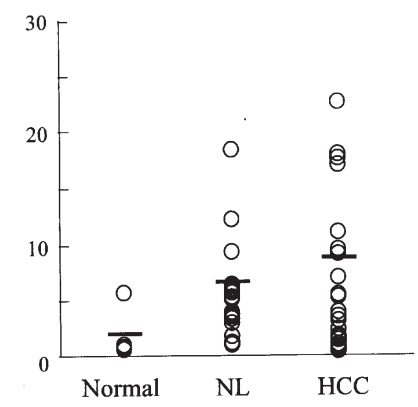

ATA2

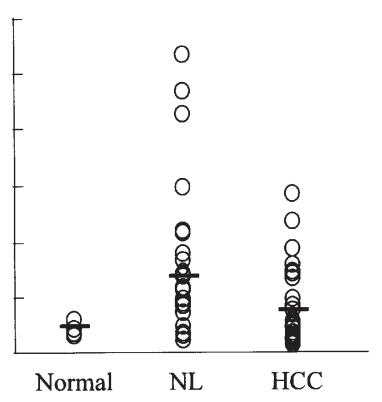

LAT1

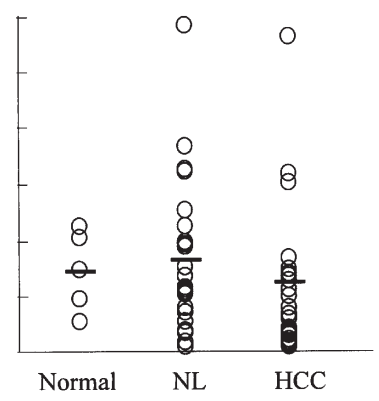

Figure 2. Expression of ATA1, ATA2, and LAT1 mRNAs in five normal livers (normal), 25 HCCs and matched non-cancerous liver tissues. Total RNA extracts from these tissues were subjected to QRT-PCR as described (16). The relative mRNA expression levels were then calculated by normalizing with a $\beta_{2}$-microglobulin control. Each circle represents a patient sample. Bars denote average expression levels. NL, non-cancerous livers; HCC, hepatocellular carcinomas.

ATA1 expression was also undetectable in the fibroblastic non-parenchymal cells. In contrast, the cytoplasmic ATA1 protein levels showed markedly increased expression levels in well-differentiated HCC and adjacent cirrhotic liver tissues (Fig. 3C, D and E). It is noteworthy also that a marked accumulation of ATA1 was observed in the micro-bile ducts of cirrhotic liver samples (Fig. 3E arrow), and on the luminal sides of pseudo-bile duct structures in well-differentiated HCC tissues (Fig. 3D arrow). High levels of cytoplasmic ATA1 were also observed in both moderately (Fig. 3F and G), and poorly differentiated (Fig. 3H and I) HCCs. We further examined the ATA1 protein levels in an additional panel of 15 HCC samples and matched non-cancerous liver tissues and detected substantial levels of expression in each of the non-cancerous liver specimens from individuals suffering from either cirrhosis or hepatitis. These expression levels were significantly elevated in five, and were similar in a further six HCCs, compared with the matched pre-cancerous livers (Table I). ATA1 expression was found to be very low in the remaining four HCC samples in this cohort (Table I). Among these tissues, no particular correlation could be found between the histological tumor grades and ATA1 protein levels. Taken together, our data show therefore that the ATA1 protein levels are markedly up-regulated in pre-cancerous and cancerous liver tissues compared with normal liver.

Functional significance of ATAl activation in HCC. Our results using both cell lines and tissues demonstrated that the expression of ATA1 was prominently activated in HCCs and also in pre-cancerous liver lesions. To assess the functional significance of ATA1 activation in $\mathrm{HCC}$, we performed siRNA knockdowns of endogenous ATAl transcripts in the HepG2 cells. To reduce the possibility of off-target effects, these cells were transfected with two independently designed ATAl-specific siRNAs, denoted HDCK and DFI, respectively. Compared to the randomized dsRNA cocktail control, both the HDCK and DFI siRNAs were found to significantly reduce the endogenous $A T A l$ transcript levels to $<10 \%$ and $<17.5 \%$, respectively, of the normal control $12 \mathrm{~h}$ after transfection (Fig. 4A). As shown in Fig. 4B, the viability of the DFI transfectants was reduced to $79.0 \%$ on day 2 , and was further reduced to $64.4 \%$ on day 3 . In a similar fashion, the cell viability of the HDCK-transfectants was reduced to 68.0 and $55.0 \%$ on days 2 and 3 , respectively. Since these two independent siRNA molecules exerted very similar effects on cell viability, we conclude that the activation of the ATAl gene in HCC cells confers a growth or survival advantage.

\section{Discussion}

Our findings indicate that the expression of amino acid transporter genes, ATA1 and ATA2, are activated in cancerousand pre-cancerous liver tissues in patients suffering from cirrhosis and/or hepatitis, but remained at lower levels in normal liver tissue from non-HCC patients. Analogous to our present observations, a neural amino acid transporter, $\mathrm{ATB}^{0}$, has also been shown previously to be overexpressed in cirrhotic liver samples (19). It has been further reported that the activity of ATA2 $(20,21)$ is adaptively up-regulated in response to physiological changes such as nutrient starvation. Since the cellular requirements for amino acids including L-arginine are increased during liver cirrhosis and fibrosis (22-24), the elevation of AT mRNAs in precancerous livers may thus also be attributable to nutrient starvation.

Our results demonstrate that the expression of ATAl and ATA2 was, in general, elevated in pre-cancerous liver tissue in patients suffering from hepatitis and/or cirrhosis compared with normal liver tissues. However, in HCC tissues, these expression patterns varied; the expression of ATAl mRNA was averagely elevated, while ATA2 mRNA was reduced in HCCs compared with pre-cancerous livers in HCC patients. These results suggest that the regulatory mechanisms underlying AT expression are quite diverse during the pre-malignant to malignant transition in liver cells. We also confirmed that ATAl mRNA was highly activated in four separate HCC cell lines cultured in standard growth media, suggesting that the elevated expression of ATAl is not simply due to an adaptive response such as nutrient starvation in HCC cells but rather a tumor-specific mechanism. System A amino acid transporters mediate the $\mathrm{Na}^{+}$-coupled transport of neutral amino acids including alanine, serine and glutamine $(1,25)$. Among the three system A isoforms that have been characterized, ATA1 

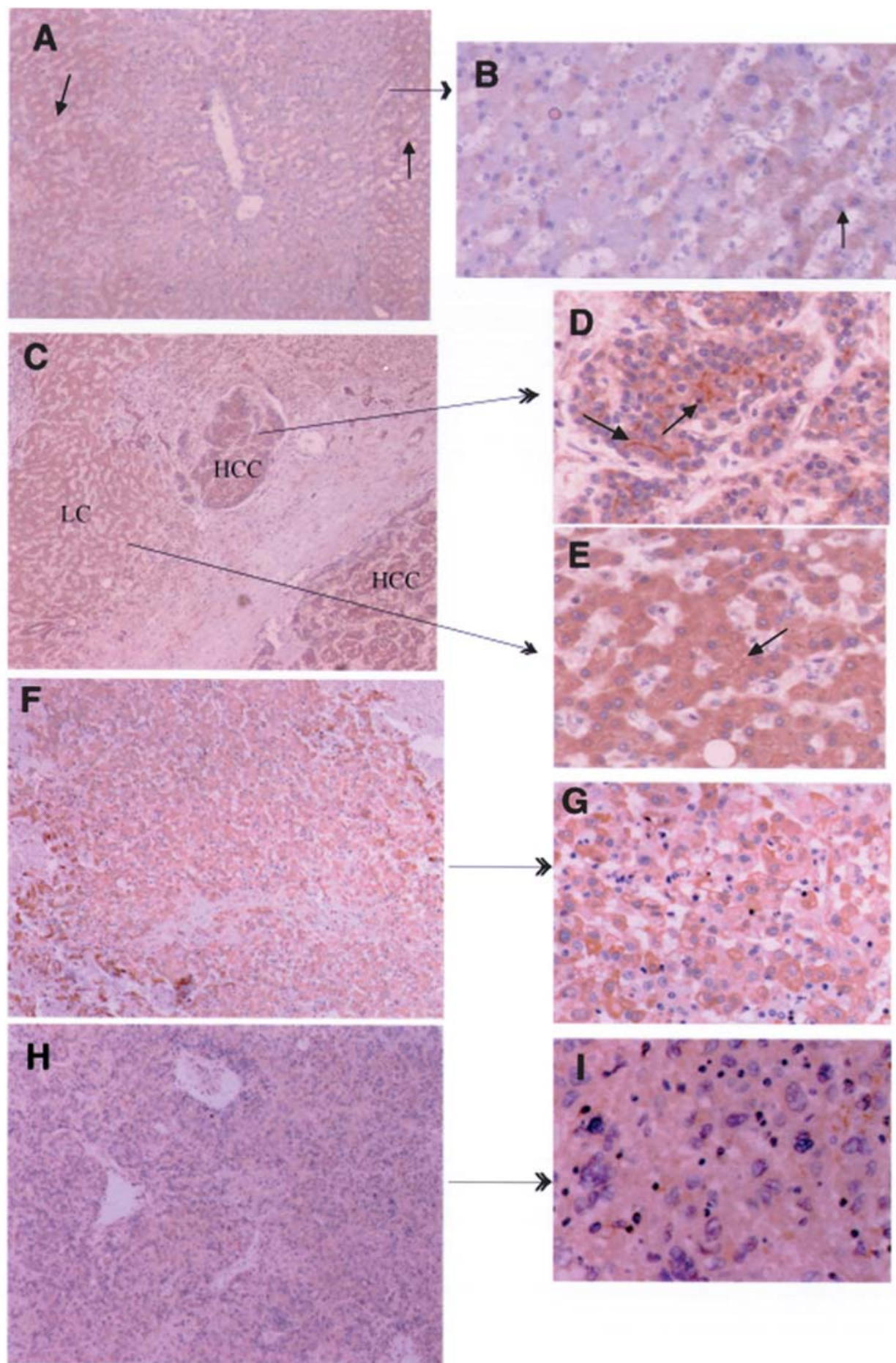

Figure 3. Immunohistochemical analysis of normal liver (A and B), well-differentiated HCC (C and D), adjacent non-cancerous liver tissue (C and E), moderately (F and $\mathrm{G}$ ), and poorly (H and I) differentiated HCCs. Sections were stained with polyclonal antibodies against ATA1; magnification x40 or x160. Representative tissues in C, D and E correspond to specimen 7; F, G to specimen 5; and H, I to specimen 11 in Table I, respectively.

has the highest affinity for glutamine (26), is strongly expressed in glutamatergic neurons, and has been suggested to participate in the cycling of glutamate and glutamine transportation between astrocytes and glial cells $(7,27)$. In normal fetal and adult hepatocytes, the uptake of glutamine is mainly mediated by system N (7) or, potentially by ATA3 as mentioned above. Although, ATA1 is primarily expressed in neurons, and not in normal liver (7), our results demonstrate that its expression is significantly elevated both in HCC tissues and HCC cell lines. In this regard, it is noteworthy that since tumors use glutamine as a primary metabolic fuel and nitrogen source $(28,29)$, a marked depletion of plasma glutamine occurs in patients with liver cancer (30). Hence, such an ectopic activation of ATA1 may be a compensatory amino acid uptake mechanism that confers a survival advantage in HCC cells.

Our histological observations demonstrate that the ATA1 protein is only marginally expressed around the periportal area in the normal liver but that its cytoplasmic expression is significantly enhanced both in pre-malignant and malignant liver lesions. Interestingly, a marked accumulation of ATA1 
A

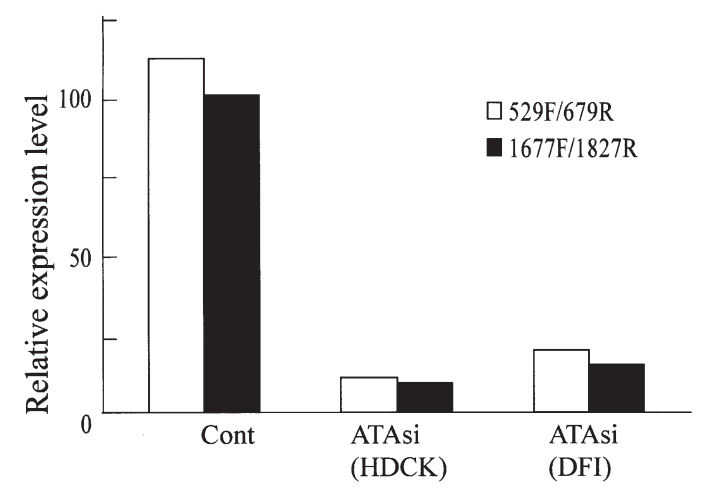

B

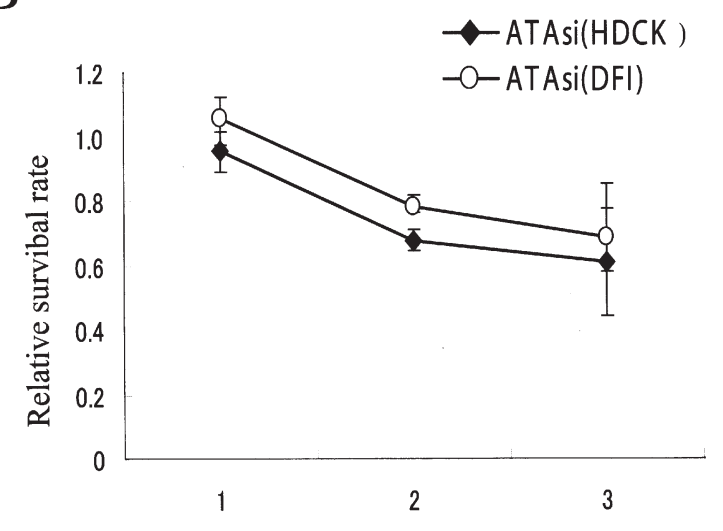

Day

Figure 4. (A) Reduced expression of endogenous ATA1 transcripts in the HepG2 cells transfected with the ATA1-specific siRNAs, HDCK and DFI. ATA1 expression was independently examined using two different specific primer pairs, 529F/679R and 1677F/1827R (see Materials and methods). (B) An XTT assay of the relative survival rates of ATA1-specific siRNA transfectants compared with randomized dsRNA transfected cells (Ordinate). (Abscissa) Days after cells were seeded. The means \pm SD for triplicate results are shown.

was also observed in micro-bile ducts in pre-cancerous cirrhotic liver and also on the luminal side of the pseudo-bile duct structure in HCC tissues. However, the functional significance of the localization of ATA1 has yet to be elucidated.

Our knockdown experiments using anti-ATAl siRNAs successfully reduced the expression of endogenous ATAl transcripts to $<18 \%$ of the control cell levels. These siRNA experiments also reduced the viability of HepG2 cells, suggesting that the ATA1 molecule is a requisite for tumor survival. However, the viability of siRNA-treated HepG2 cells still remained higher than we had expected $(>55 \%)$ given the significantly reduced mRNA levels $(<17.5 \%)$. HCC cells may thus have compensatory mechanisms for specific amino acid uptake. In fact, it is known that a neutral amino acid transporter, $\mathrm{ATB}^{0}$ is activated in $\mathrm{HCC}$ cells and promotes glutamine-dependent tumor growth (19).

In conclusion therefore, future investigations that are designed to further understand both the functional significance and the regulatory mechanisms underlying amino acid transporters could provide diagnostic and therapeutic insights into hepatocarcinogenesis.

\section{References}

1. McGivan JD and Pastor-Anglada M: Regulatory and molecular aspects of mammalian amino acid transport. Biochem J 299: 321-334, 1994.

2. Kim DK, Kanai Y, Choi HW, Tangtrongsup S, Chairoungdua A, Babu E, Tachampa K, Anzai N, Iribe Y and Endou H: Characterization of the system L amino acid transporter in T24 human bladder carcinoma cells. Biochim Biophys Acta 1565: 112-121, 2002.

3. Lin J, Raoof DA, Thomas DG, Greenson JK, Giordano TJ, Robinson GS, Bourner MJ, Bauer CT, Orringer MB and Beer DG: L-type amino acid transporter-1 overexpression and melphalan sensitivity in Barrett's adenocarcinoma. Neoplasia 6: 74-84, 2004.

4. Yoon JH, Kim YB, Kim MS, Park JC, Kook JK, Jung HM, Kim SG, Yoo H, Ko YM, Lee SH, Kim BY, Chun HS, Kanai Y, Endou $\mathrm{H}$ and Kim do K: Expression and functional characterization of the system L amino acid transporter in KB human oral epidermoid carcinoma cells. Cancer Lett 205: 215-226, 2004.

5. Kim DK, Ahn SG, Park JC, Kanai Y, Endou H and Yoon JH: Expression of L-type amino acid transporter 1 (LAT1) and 4F2 heavy chain (4F2hc) in oral squamous cell carcinoma and its precusor lesions. Anticancer Res 24: 1671-1675, 2004.

6. Campbell WA and Thompson NL: Overexpression of LAT1/ CD98 light chain is sufficient to increase system L-amino acid transport activity in mouse hepatocytes but not fibroblasts. J Biol Chem 276: 16877-16884, 2001.

7. Varoqui H, Zhu H, Yao D, Ming H and Erickson JD: Cloning and functional identification of a neuronal glutamine transporter. J Biol Chem 275: 4049-4054, 2000.

8. Sugawara M, Nakanishi T, Fei YJ, Huang W, Ganapathy ME, Leibach FH and Ganapathy V: Cloning of an amino acid transporter with functional characteristics and tissue expression pattern identical to that of system A. J Biol Chem 275: 16473-16477, 2000.

9. Hatanaka T, Huang W, Ling R, Prasad PD, Sugawara M, Leibach FH and Ganapathy V: Evidence for the transport of neutral as well as cationic amino acids by ATA3, a novel and liver-specific subtype of amino acid transport system A. Biochim Biophys Acta 1510: 10-17, 2001.

10. Freeman TL, Thiele GM, Tuma DJ, Machu TK and Mailliard ME: ATA2-mediated amino acid uptake following partial hepatectomy is regulated by redistribution to the plasma membrane. Arch Biochem Biophys 400: 215-222, 2002.

11. Freeman TL and Mailliard ME: Posttranscriptional regulation of ATA2 transport during liver regeneration. Biochem Biophys Res Commun 278: 729-732, 2000.

12. Bhat HK and Vadgama JV: Role of estrogen receptor in the regulation of estrogen induced amino acid transport of System A in breast cancer and other receptor positive tumor cells. Int J Mol Med 9: 271-279, 2002.

13. Dudeck KL, Dudenhausen EE, Chiles TC, Fafournoux P and Kilberg MS: Evidence for inherent differences in the system A carrier from normal and transformed liver tissue. Differential inactivation and substrate protection in membrane vesicles and reconstituted proteoliposomes. J Biol Chem 262: 12565-12569, 1987.

14. Wheatley DN, Scott L, Lamb J and Smith S: Single amino acid (arginine) restriction: growth and death of cultured HeLa and human diploid fibroblasts. Cell Physiol Biochem 10: 37-55, 2000.

15. Ohkura S, Kondoh N, Hada A, Arai M, Yamazaki Y, Sindoh M, Takahashi M, Matsumoto I and Yamamoto M: Differential expression of the keratin-4, $-13,-14,-17$ and transglutaminase 3 genes during the development of oral squamous cell carcinoma from leukoplakia. Oral Oncol 41: 607-613, 2005.

16. Arai M, Kondoh N, Imazeki N, Hada A, Hatsuse K, Kimura F, Matsubara O, Mori K, Wakatsuki T and Yamamoto M: Transformation-associated gene regulation by ATF6alpha during hepatocarcinogenesis. FEBS Lett 580: 184-190, 2006.

17. Matsuo H, Kanai Y, Kim JY, Chairoungdua A, Kim DK, Inatori J, Shigeta Y, Chaekuntode S, Achampa K, Choi HW, Babu E, Fukuda J and Endou H: Identification of a novel $\mathrm{Na}^{+}$independent acidic amino acid transporter with structural similarity to the member of a heterodimeric amino acid transporter family associated with unknown heavy chains. J Biol Chem 277: 21017-21026, 2002.

18. Hisano S, Haga H, Li Z, Tatsumi S, Miyamoto KI, Takeda E and Fukui Y: Immunohistochemical and RT-PCR detection of $\mathrm{Na}^{+}$-dependent inorganic phosphate cotransporter $(\mathrm{NaPi}-2)$ in rat brain. Brain Res 772: 149-155, 1997. 
19. Bode BP, Fuchs BC, Hurley BP, Conroy JL, Suetterlin JE, Tanabe KK, Rhoads DB, Abcouwer SF and Souba WW: Molecular and functional analysis of glutamine uptake in human hepatoma and liver-derived cells. Am J Physiol Gastrointest Liver Physiol 283: G1062-G1073, 2002.

20. Alfieri RR, Bonelli MA, Petronini PG, Desenzani S, Cavazzoni A, Borghetti AF and Wheeler KP: Hypertonic stress and amino acid deprivation both increase expression of mRNA for amino acid transport system A. J Gen Physiol 125: 37-39, 2005.

21. Gazzola RF, Sala R, Bussolati O, Visigalli R, Dall'Asta V, Ganapathy V and Gazzola GC: The adaptive regulation of amino acid transport system A is associated to changes in ATA2 expression. FEBS Lett 490: 11-14, 2001.

22. Kim WH, Park H, Yun C, Cho H, Kim S, Paik WK, Jeon SH and Lee JH: Mixture of N-carbamoyl-L-glutamate plus Larginine can protect rats with liver cirrhosis from acute ammonia intoxication. J Hepatol 35: 719-725, 2001.

23. Nanji AA, Jokelainen K, Lau GK, Rahemtulla A, Tipoe GL, Polavarapu R and Lalani EN: Arginine reverses ethanol-induced inflammatory and fibrotic changes in liver despite continued ethanol administration. J Pharmacol Exp Ther 299: 832-839, 2001.
24. Zimmermann A, Bachmann C and Baumgartner R: Severe liver fibrosis in argininosuccinic aciduria. Arch Pathol Lab Med 110: 136-140, 1986.

25. Bode BP: Recent molecular advances in mammalian glutamine transport. J Nutr 131 (suppl): S2475-S2485, 2001.

26. Bode BP, Kaminski DL, Souba WW and Li AP: Glutamine transport in isolated human hepatocytes and transformed liver cells. Hepatology 21: 511-520, 1995.

27. Weiss MD, Derazi S, Rossignol C, Varoqui H, Erickson JD, Kilberg MS and Anderson KJ: Ontogeny of the neutral amino acid transporter SAT1/ATA1 in rat brain. Brain Res Dev Brain Res 143: 151-159, 2003.

28. Medina MA, Sanchez-Jimenez F, Marquez J, Rodriguez Quesada A and Nunez de Castro I: Relevance of glutamine metabolism to tumor cell growth. Mol Cell Biochem 113: 1-15, 1992.

29. Chen MK, Espat NJ, Bland KI, Copeland EM III and Souba WW: Influence of progressive tumor growth on glutamine metabolism in skeletal muscle and kidney. Ann Surg 217: 655-667, 1993.

30. Hirayama C, Suyama K, Horie Y, Tanimoto K and Kato S Plasma amino acid patterns in hepatocellular carcinoma. Biochem Med Metab Biol 38: 127-133, 1987. 